\title{
Cosmic-ray acceleration and gamma-ray signals from radio supernovæ
}

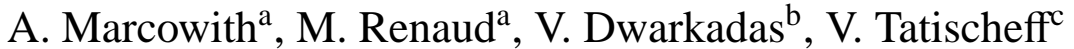 \\ ${ }^{a}$ Laboratoire Univers et particules de Montpellier, Université Montpellier II/CNRS, place E. Bataillon, cc072, 34095 Montpellier, France \\ ${ }^{b}$ Department of Astronomy and Astrophysics, University of Chicago, Chicago, Illinois, 60637, USA \\ ${ }^{c}$ Centre de Sciences Nucléaires et de Sciences de la Matière, IN2P3/CNRS and Univ Paris-Sud, 91405 Orsay, France
}

\begin{abstract}
Core collapse supernovae ( $\mathrm{SNe}$ ) are among the most extreme events in the universe. The are known to harbor among the fastest (but non- or mildly-relativistic) shock waves. Once it has crossed the stellar atmosphere, the SN blast wave expands in the wind of the massive star progenitor. In type IIb SNe, the progenitor is likely a Red SuperGiant (RSG) star which has a large mass loss rate and a slow stellar wind producing a very dense circumstellar medium. A high velocity shock and a high density medium are both key ingredients to initiate fast particle acceleration, and fast growing instabilities driven by the acceleration process itself. We have reanalyzed the efficiency of particle acceleration at the forward shock right after the SN outburst for the particular case of the well-known SN 1993J. We find that plasma instabilities driven by the energetic particles accelerated at the shock front grow over intraday timescales. This growth, and the interplay of non-linear process, permit a fast amplification of the magnetic field at the shock, that can explain the magnetic field strengths deduced from the radio monitoring of the source. The maximum particle energy is found to reach 1-10 PeV depending on the instability dominating the amplification process. We derive the time dependent particle spectra and the associated hadronic signatures of secondary particles (gamma-ray, leptons and neutrinos) arising from proton proton interactions. We find that the Cherenkov Telescope Array (CTA) should easily detect objects like SN 1993J in particular above $1 \mathrm{TeV}$, while current generation of Cherenkov telescopes such as H.E.S.S. could only marginally detect such events. The gamma-ray signal is found to be heavily absorbed by pair production process during the first week after the outburst. We predict a low neutrino flux above $10 \mathrm{TeV}$, implying a detectability horizon with a KM3NeT-type telescope of $1 \mathrm{Mpc}$ only. We finally discuss the essential parameters that control the particle acceleration and gamma-ray emission in other type of SNe.
\end{abstract}

Keywords: Supernovae, Shock Acceleration, Gamma-ray and neutrino emission.

\section{Introduction \\ It is widely accepted that SuperNova Remnants} (SNRs) are likely the sources of Cosmic Rays (CR) in our Galaxy up to energies corresponding to the so-called CR "knee" at $3 \mathrm{PeV}$ for the proton component (e.g. see [1]). However, an important question remains: if SNRs do produce $\mathrm{PeV}$ particles, when does this acceleration occur in the SNR lifetime? Another issue is that in order to explain the whole CR spectrum one has to consider particles to be accelerated not only up the knee but up to a few hundred PeV, where an extragalactic component should start taking over. Hence, only a restricted number of Galactic sources should be able to produce such energetic particles following the confinement criterium (see [2]) stating that the Larmor radius of the particle should match the source size. Either extended sources with standard interstellar medium magnetic field values or more compact sources but with more intense magnetic fields could fulfill such a criterium. In the first category one finds the massive star clusters and their superbubbles [3, 4, 5] while the second category comprises young supernova remnants [6]. This latter work has already pointed out the possibility to produce multi-PeV CR energies in very young SNRs rather than in more evolved objects entering the Sedov phase. It is then very important to propose accurate modeling of the time history of CR 
acceleration in a SNR that can be tested by forthcoming instruments such as the Cherenkov Telescope Array (CTA).

This work addresses the issue of particle acceleration and gamma-ray radiation in the so-called wind $\mathrm{SNe}$ arising from massive star progenitors and evolving in the dense CSM. It provides observationally testable modeling of these early stages. The novelty here is that we consider particle acceleration right after the outburst when the radio luminosity is close to its maximum. The discussion in this paper is mainly focused on one particular well-monitored object: SN 1993J. We will review the main results of radio observations and their modeling in $\$ 2$. Some perspectives for other types of SNe will be discussed in $\$ 4$. We have adapted recent models of magnetic field amplification to the case of SN 1993J in $\$ 2.1$ High magnetic field means likely higher CR energies as stated above. This will be examined in $\$ 2.2$ We will detail the modeling of the different particle distribution species in radio $\mathrm{SNe}$ in $\$ 2.3$. Once the particle distribution has been derived we will address the multiwavelength radiation (in $\$ 3.2$ ) and the neutrino (in $\$ 3.3$ ) signal that would have been detected from SN 1993J by modern instruments if they were operational at the time of the SN outburst. Our main prediction concerning the gamma-ray radiation is detailed in $\$ 3.1$ and a conclusion is given in $\$ 5$

\section{A case study: SN 1993J}

Our model is based on the work proposed by [7] (hereafter T09) to account for particle acceleration in SN 1993J. This SN is a type IIb SN resulting from the explosion of a massive star with an initial mass between 13 to $20 M_{\odot}$ (in the case the explosion occurred in a binary system) or 20 to $30 M_{\odot}$ in the case of an isolated star [8]. The star then evolved into a Red SuperGiant (RSG) phase with a mass loss rate of $\sim 3.8 \times 10^{-5} M_{\odot} / \mathrm{yr}$ and a slow wind velocity of $u_{w} \sim 10 \mathrm{~km} / \mathrm{s}$ (see T09 for the derivation of these parameters). These values imply that in case of a constant mass loss rate the density in circumstellar medium (CSM) scales as:

$$
n_{\text {circ }}=\frac{\dot{M}(1+2 X)}{4 \pi r^{2} u_{w} m_{H}(1+4 X)},
$$

where $X=0.1$ is the Helium fraction and $m_{H}$ is the hydrogen atom mass. With the above parameters the effective density downstream of the forward shock is typically $n_{\text {eff }} \simeq 4 \times 10^{9} \mathrm{~cm}^{-3}$ at the time of outburst (here we have accounted for a shock compression ratio of 4 ).
The radius of the shock at the outburst $r \simeq 3.5 \times 10^{14} \mathrm{~cm}$ (see T09) has been deduced from the derivation of the radio expansion. The shock is expected to propagate into a fully ionized medium [9].

T09 proposed a scenario based on the radio follow-up observations of SN 1993J over a period covering 3100 days after the SN outburst. The synchrotron model provides fits to light curves at 6 different radio wavelengths, including the effects of synchrotron-self-absorption and free-free absorption. The effect on the radio signal attenuation by the homogeneous CSM matter, as well as the presence of clumps, has been taken into account in the modeling. This resulted in the following time dependence of the averaged total magnetic field from the synchrotron emitting shell:

$$
\langle B\rangle \simeq[2.4 \pm 1 \mathrm{G}] \times\left(\frac{\mathrm{t}}{100 \text { days }}\right)^{-1.16 \pm 0.20},
$$

which points towards a magnetic field of the order of $500 \mathrm{G}$ after 1 day. This field is consistent with observations [17]. The above magnetic field is only an averaged value over the synchrotron shell and does not represent the magnetic field produced at the forward shock necessarily. In the context of the model of CR driven instabilities detailed afterward, we will identify this field with the one obtained in the post shock gas of the forward shock front. The magnetic field in the upstream medium can hence be derived from the generalized Rankine-Hugoniot conditions at the shock front accounting for the CR back-reaction and the turbulent magnetic field heating at the shock precursor (see details in [10, 11]).

\subsection{Magnetic field amplification (MFA)}

Eq. (2) clearly shows that the magnetic field is still much larger than typical field expected in the wind of massive stars at such distance. For comparison the equipartition magnetic field in the progenitor wind is [12]:

$$
\begin{aligned}
B_{e q}= & \frac{\left(\dot{M} u_{w}\right)^{1 / 2}}{r} \\
& \simeq[2.5 \mathrm{mG}] \times \dot{\mathrm{M}}_{-5}^{1 / 2} \times \mathrm{u}_{\mathrm{w}, 10}^{1 / 2} \times \mathrm{r}_{16}^{-1},
\end{aligned}
$$

about one thousand times less than the value derived in Eq 2. The solutions of particle acceleration by non-linear diffusive shock acceleration model (based on the approach of [13]) proposed in T09 are not strongly modified by the CR pressure. This means that the sub-shock compression ratio should not be far from 4 . Then the compression of the magnetic field 
given by Eq. (3) cannot explain the field value given by Eq. (2) (see also the discussion in [12]). We conclude and assume from now that a strong magnetic field amplification process is at work at the forward shock front and it is driven by the diffusive shock acceleration (DSA) of hadrons.

We now discuss in this framework the possible mechanisms that can produce such an amplification. Note that for our purpose it is simply required that there exists a fast instability to produce the requested MFA at scales where the CRs are efficiently scattered. The magnetic field amplification at fast shocks in young SNR is likely connected with the presence of energetic particles (likely to become CRs) moving in front of the shock and producing a precursor. We can make a distinction among several types of instabilities: the instabilities produced by the pressure gradient of cosmic rays, the instabilities produced by the streaming of cosmic rays (see [14] for a recent review). The magnetic field can be amplified due to the presence of background turbulence, and further amplified at the shock front.

\subsubsection{Streaming driven instabilities}

The streaming of cosmic rays ahead the shock front produces magnetic fluctuations that have two different different regimes. The streaming modes can either be in resonance $(\mathrm{R})$ with the energetic particles, i.e. in the high-energy limit they have a wave-number such that $k \sim \mathrm{r}_{\mathrm{L}}^{-1}$ or they can be non-resonant (NR) with a wave-number much larger than $\mathrm{r}_{\mathrm{L}}^{-1}[15,16,19]$. The NR modes grow the fastest especially in the regime of fast shocks (see [20]). As already discussed in T09 within the conditions that prevails for IIb SN, the NR modes grow and produce magnetic fluctuations over intra-day timescales; we get from the time of the outburst using the shock parameters described above a NR mode growth time of:

$$
\begin{aligned}
\tau_{N R-s t}= & {[0.16 \text { day }] \times\left(\frac{\phi / 15}{\left(\xi_{\mathrm{CR}} / 0.05\right) \mathrm{u}_{\mathrm{sh}, 93 \mathrm{~J}}^{3} \sqrt{n_{93 J}}}\right) } \\
& \times E_{P e V} t_{\text {days }}^{1.17} .
\end{aligned}
$$

Here we have assumed that the CR distribution scales as $p^{-4}$ over more than 6 orders of magnitude producing $\phi=\ln \left(p_{\max } / p_{\text {inj }}\right)=15$ and that $\xi_{C R}=0.05$ namely that $5 \%$ of the fluid kinetic energy is imparted into energetic particles. Here also, $p_{\max }\left(p_{i n j}\right)$ is the maximum (the injected) particle momentum. The growth timescale has to be shorter than the advection timescale towards the shock $\tau_{a d v}=\kappa / V_{s h}^{2}$. The advection timescale is calcu- lated for a coefficient $\kappa=\eta \kappa_{B}>\kappa_{B}$ taken in the background magnetic field (Eq 3 ) and $\kappa_{B}=c r_{L} / 3$. We find:

$$
\tau_{a d v}=[0.24 \text { day }] \times \eta \mathrm{E}_{\mathrm{PeV}} \times \mathrm{t}_{\mathrm{day}}^{1.17} .
$$

The condition $\tau_{N R-s t}<\tau_{a d v}$ is necessary for the instability to develop and for CR being confined around the shock, but it is not sufficient as the magnetic fluctuations produced by the instability uncovered by Bell [16] produces small scale perturbations. The wave number corresponding to the maximum growth rate is [19] $k_{\text {Gmax }} r_{\text {Lmax }} \simeq 4 \times 10^{6}$ for the conditions prevailing in SN 1993J. The acceleration and confinement of energetic particles up to a few $\mathrm{PeV}$ requires magnetic fluctuations to be generated at resonant scales $k r_{L} \simeq 1$, as the NR growth rate scales as $k^{1 / 2}$ the magnetic field at the scale of interest grows over a timescales about 2000 times larger than the one obtained in Eq. 4 It should be noted that if the background magnetic field is purely toroidal we may expect $\eta<1$. In that case, the instability may not have time to develop. There are no measurements that point towards a particular topology of the magnetic field in the wind of different type of evolved stars [18]. The winds are themselves very likely inhomogeneous, subject to turbulent motions that can introduce some tangling effects in the background magnetic field even if on the scale of the wind it verifies $\mathrm{Eq} 3$ We postpone this issue for further work and admit $\eta \geq 1$ in this work.

Longer wavelengths can be generated by different means. The R instability can build up over the NR one as demonstrated in [20] with a ratio of the two magnetic energies reaching $\sqrt{\xi_{C R} c / V_{s h}} \sim 0.95$ in our case. The growth timescale for the $\mathrm{R}$ instability is however longer than the rate given by Eq. 4. We have (see [19]) $\tau_{R-s t} \simeq \sqrt{\pi \sigma / 8} / r_{\text {Lmax }}$ with $\sigma \sim 3 \times 10^{16} \mathrm{~cm}^{2} / \mathrm{s}^{2}$ in the conditions that prevail for SN 1993J. This leads to a growth rate $\tau_{R-s t} \simeq 16 \tau_{N R-s t}$ at $1 \mathrm{PeV}$. Other long wavelength instabilities have to be considered. The NR instability can be driven to non-linear stages and produce large wave numbers. A typical timescale of nonlinear saturation of the magnetic field is about $5 \times \tau_{N R, s t}$ [6]. Recently [21] proposed a ponderomotive instability that builds up on the magnetic fluctuations by the NR streaming instability. We can evaluate the growth timescale of such long-wavelength modes:

$$
\begin{array}{r}
\tau_{L W}=[0.29 \text { day }] \times \sqrt{\left(\frac{\phi / 15}{\xi_{C R} / 0.05}\right)} \\
\times \frac{1}{\sqrt{u_{s h, 93 J}^{3} A_{10}}} \times E_{P e V} t_{\text {days }} .
\end{array}
$$


The parameter $A=B_{N R}^{2} / B_{0}^{2}>1$ is the level of magnetic energy produced by the small scale instability with respect to the background CSM and is normalized to 10 (which is rather underestimated in our case). This timescale is sufficiently short to produce long wavelengths on timescales shorter than $\tau_{a d v}$ for $k r_{L, \max } \simeq 1$.

\subsubsection{Turbulently driven instabilities}

The stellar wind of RGB star is subject to strong fluid instabilities and is likely turbulent [22]. Turbulent density and magnetic fluctuations can have a strong impact over the SN shock and lead to magnetic field amplification [23, 24]. Note finally that in the latter work the presence of CR is not even necessary to produce the magnetic field amplification. In that case the magnetic field growth time is controlled by the coherence length of the turbulent spectrum $\mathrm{L}$ and by the fluid velocity $u_{s h}$. In the conditions of SN1993J, $L / u_{s h} \sim$ [0.3 year] $\mathrm{L}_{0.01 \mathrm{pc}} / \mathrm{u}_{\mathrm{sh}, 93 \mathrm{~J}}$ hence the magnetic field has to grow over a fraction of $10^{-2}$ of this timescale.

\subsection{Maximum cosmic ray energies}

At very early timescales after the outburst, the likely maximum energy limitation is provided by the SNR age. The maximum energy is hence fixed balancing the age with the acceleration time $\tau_{a c c}=g(r) \kappa_{u} / u_{s h}^{2}$, with $g(r)$ can be expressed with the shock compression ratio as $g(r)=3 r /(r-1) \times\left(\kappa_{d} / \kappa_{u} r+1\right)$. The ratio of the downto upstream diffusion coefficient depends on the magnetic field orientation with respect to shock (1 in a parallel shock, $1 / r$ in a perpendicular shock). As discussed above we admit $\kappa_{d}=\kappa_{u} / \sqrt{11}$ in our case. This corresponds to a completely tangled magnetic field whose tangential component is compressed by a factor 4 (see [11]). We obtain a maximum energy expressed in $\mathrm{PeV}$ units:

$$
E_{\text {max }, \text { age }, P e V} \simeq \frac{12.3}{\eta g(r)} \times\left(1-t_{\text {day }}^{-0.17}\right) .
$$

But rapidly (see $\$ 2.1$ the streaming instability amplifies the magnetic field and non-linear process produces a magnetic field at saturation. The typical saturation value expected from the NR instability (see [16]) is:

$$
B_{\text {sat }}=[16 \text { Gauss }] \times \sqrt{\frac{\xi_{C R} / 0.05}{\phi / 15}} \times \mathrm{t}_{\text {days }}^{-1} .
$$

This value, as already remarked by T09, is in agreement (within a factor of 2) with the magnetic field derived in the upstream medium from $\mathrm{Eq} 2$ using a compression ratio $r=4$. If only the NR instability is at work building the magnetic field hence the maximum particle energy is fixed by a condition over the CR areal charge [6] that produces $\int \tau_{N R-s t}^{-1} d t=6.8$ (a little bit larger than what Bell et al. have considered). The latter value corresponds to the amplification of the equipartition magnetic field to the value deduced from radio observations. In that case:

$$
E_{\text {max }, N R, P e V} \sim 1 \times t_{\text {day }}^{-0.17} .
$$

But if long wavelengths fluctuations are produced by the mean of the ponderomotive instability the maximum energy is fixed by geometrical losses. The maximum energies are obtained with a diffusion coefficient expressed in the amplified field and compared to $\eta_{e s c} R_{s h} u_{s h}$. In order to derive a time dependence of the maximum energy the time dependence of the amplified field has to be specified. The non-linear regime of the ponderomotive instability has not yet been fully explored (see however some attempts in [25]). In consequence, we rely on the estimate given in Eq. 8 to fix such a dependence. This gives:

$$
E_{\text {max }, L W, P e V} \sim 55\left(\frac{\eta_{\text {esc }}}{0.1}\right) \times t_{\text {day }}^{-0.34} .
$$

Apart from the question of time dependence of the saturated magnetic field, this value is optimistic also since the magnetic field experienced by the highest energies is likely lower than $B_{\text {sat }}[26]$. The maximum cosmic ray (proton) energy is at any given time the minimum of the above limits. In all cases, $\mathrm{PeV}$ energies at least can be reached at early timescales after the outburst. These energies can be probed using a gamma-ray telescope sensitive above a few hundred $\mathrm{TeV}$.

In our model exposed hereafter the maximum electron energy has also been calculated and is found to be fixed by the radiative losses (here synchrotron) losses.

\subsection{Cosmic-ray spectral evolution}

We basically follow the prescription of T09 regarding the time evolution of the CR energy content (see their Eq. 50). The proton particle spectrum is assumed to follow a power-law with a spectral index $s=2$ and an exponential cutoff at $E_{\max }(t)$, the maximum particle energy as discussed in $\$ 2.2$ In the case of secondary electrons and positrons produced in the proton-proton interactions, we solved a one-zone energy equation to calculate the time evolution of their energy distribution $N(E, t)$ :

$$
\partial_{t} N(E, t)+\partial_{E}(\dot{L}(E) N(E, t))=Q(E, t)
$$

The term $\dot{L}$ includes the radiative (synchrotron) losses affecting the secondary electrons and positrons in the the post-shock region. 


\section{Multi-wavelength and multi-messenger spectra}

\subsection{Gamma-Ray production}

In this section we derive the multi-wavelength time dependent spectra to be expected in the context of MFA driven by the instabilities discussed in section $\$ 2.1$ First, we restricted our analysis solely to the protonproton interactions. Inverse Compton or bremsstrahlung radiation have been found to be negligible in the GeV$\mathrm{TeV}$ gamma-ray domain explored here. Especially the Inverse Compton process is highly disfavored due to the strong magnetic field at the forward shock. Gammarays once produced can be absorbed by different soft photon fields to produce electron-positron pairs. The main photon source is the $\mathrm{SN}$ photosphere which has been well described in the case of SN 1993J by [27].

\subsubsection{Gamma-gamma absorption}

The gamma-gamma absorption process is complicated by the fact that the soft photon distribution produced by supernova photosphere looks more and more anisotropic by the gamma-rays generated at the forward shock as time goes on. We have performed a full calculation of the gamma-gamma opacity $\tau_{\gamma \gamma}$ including the geometrical effects due to the anisotropic interaction (Renaud et al. 2014 in prep). The final gamma-ray flux is hence the unabsorbed flux $F_{v, u n}$ times an attenuation factor $\exp \left(-\tau_{\gamma \gamma}\left(E_{\gamma}\right)\right)$. The gamma-gamma absorption effect is strong just after the outburst as the interaction in nearly isotropic and matches the derivation proposed in T09, but thereafter drops as the ratio of the forward shock radius to the photosphere radius reaches $\sim 3$, which happens after $\sim 5$ days. The anisotropic absorption then boosts the gamma-ray signal one may expect with respect to the fully isotropic case considered by T09.

\subsubsection{Cherenkov Telescope Array and H.E.S.S. de- tectability}

The time dependent gamma-ray spectra in the veryhigh energy ( $100 \mathrm{GeV}<\mathrm{E}<100 \mathrm{TeV})$ gamma-ray domain is displayed in figure 3.1.2 (notice that the figures will be in colors only in the online version, whereas they will be $b / w$ in print.) We find that a source like SN 1993J would be easily (resp. marginally) detected by CTA (resp. H.E.S.S.), in particular above $1 \mathrm{TeV}$, in $20 \mathrm{~h}$ of observing time. We predict that in this type of object the best time window to detect a gamma-ray signal is between a week and a month after the outburst. Prior to a week the source is optically thick to gamma-rays, but the gamma-gamma opacity decreases rapidly due to anisotropic effects. After a month the gamma-ray signal

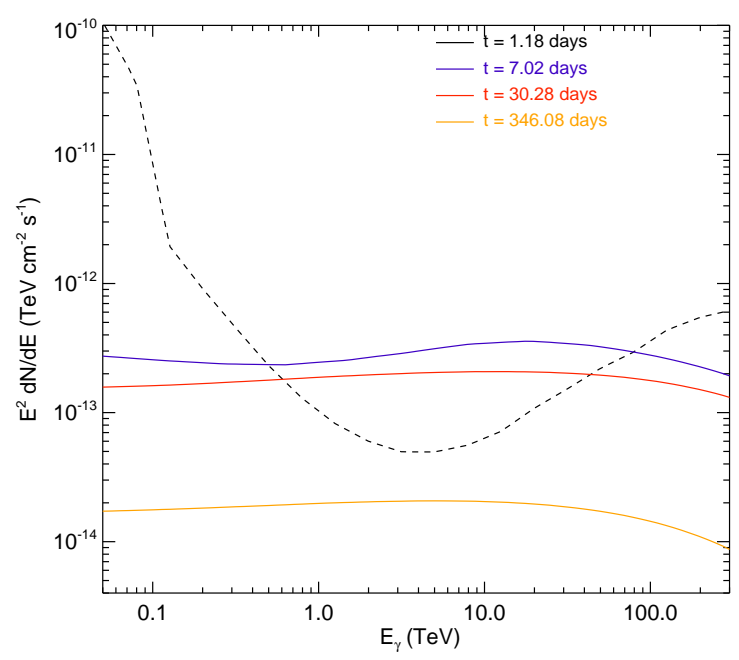

Figure 1. Time dependent spectra in the CTA energy domain at four different times after the outburst. CTA sensitivity in 50h is shown with dotted lines. At $\mathrm{t}=1.18$ days, the source is optically thick to gamma rays, which leads to a very low flux not seen in the Figure.

becomes too faint to be detected as the forward shock moves into a less dense wind medium.

\subsection{Radiation by secondaries}

The emission produced by the secondary particles issued from the pion production process is displayed in Figure 3.2. Note that the radiation of the electronpositron pairs produced in the electromagnetic cascade triggered by the gamma-rays is not included in the calculations, but this can only modify the secondary flux within the first days after the outburst when the source is optically thick to gamma-rays. We find that the flux produced by the secondaries is below the level of the observed radio and X-ray data, consistent with our previous expectation.

\subsection{Neutrino signal}

Neutrinos are also by-products of pion production. The expected flux of neutrinos to be detected by an instrument equivalent to $\mathrm{KM} 3 \mathrm{NeT}$ is displayed in Figure 3.3 We find that at best about 0.1 neutrinos could be expected from a SN 1993J type event above $10 \mathrm{TeV}$ by summing the spectra between 1 and 30.28 days after the outburst whereas for the same integration time 0.03 background neutrinos would ve expected. Hence, in order to obtain at least one neutrino form such a source it must be at a distance of $\sim 1 \mathrm{Mpc}$ or the gamma-ray signal has to be ten times stronger. 


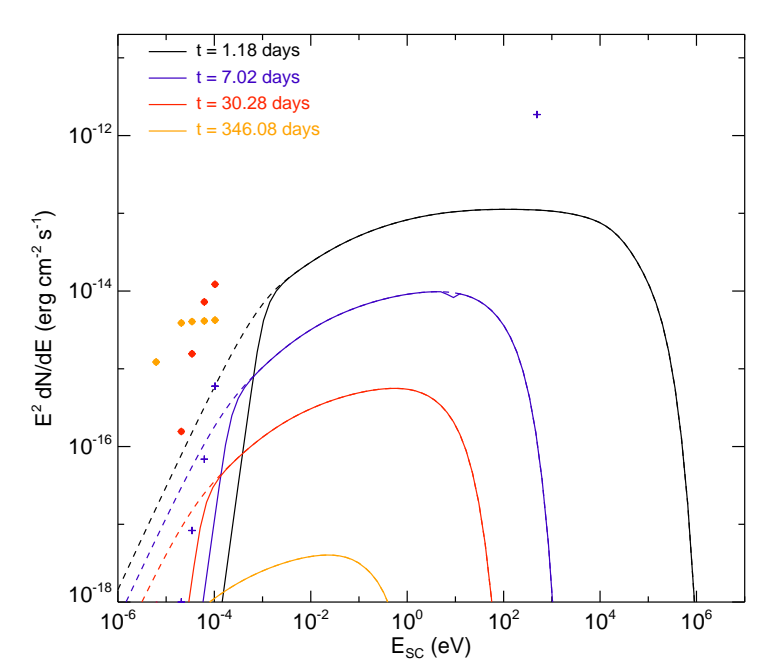

Figure 2. Time dependent spectra from electron-positron secondaries at fourth different times after the outburst. Radio data are from [28]. X-ray data are from [29] and have been taken at about 7 days after the outburst. The dotted lines represent the un-absorbed synchrotron spectra while the continuous lines include synchrotron self-absorption (see T09).

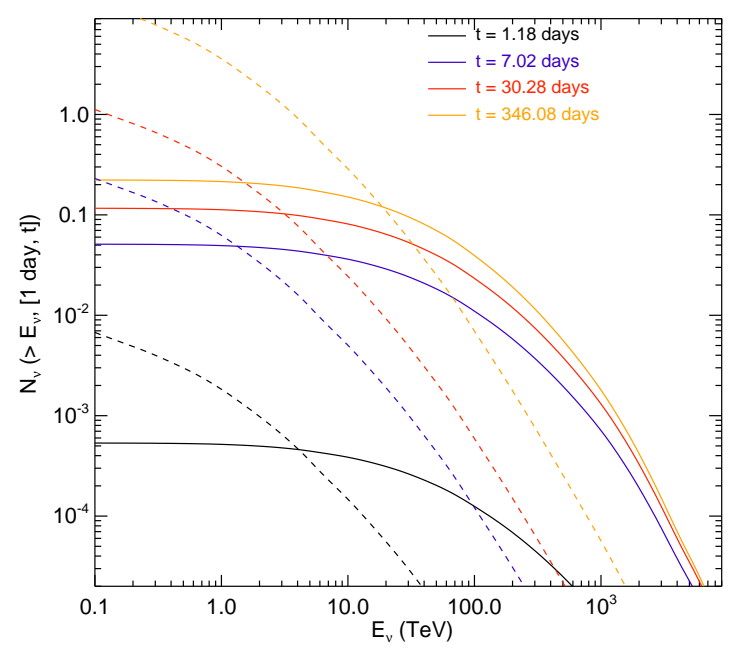

Figure 3. Time dependent neutrinos flux above an energy E expected by an instrument equivalent to KM3NeT (continuous lines) at fourth different times after the outburst. In dotted lines are displayed the atmospheric neutrino backgrounds.

\section{Discussion}

Type IIb SNe like SN 1993J are rare events. Only about $5-6 \%$ of the local core-collapse SNe have been classified in that category [30]. One of the most important parameter controlling the early gamma-ray emission from $\mathrm{SNe}$ is the ratio of the mass loss rate and the wind velocity $\dot{M} / v_{w}$. This parameter fixes the CSM medium density and the different CR driven instability growth rates. The other important parameters are: the shock velocity, the local ionization degree of the CSM matter (see [31]), the background stellar wind magnetic field and the SN peak luminosity. The shock velocity controls the growth rate of the instabilities and the acceleration timescale. The ionization degree is important for the particle acceleration efficiency and may also produces element dependent CR spectra if the ionization is incomplete for heavier elements. The background magnetic field controls partly the local magnetization and the shock obliquity. The SN luminosity controls the gamma-gamma absorption process. The effects of each these parameters deserves further investigation and will be explicitly treated in a future work.

Taking the combined effects of the above parameters into account we can examine the possibility for observations of other SN types. In terms of high ambient density, it appears that Type IIn SNe would be one of the most promising targets for gamma-ray telescopes. Although high density implies lower velocity of the shock wave, the dependence of the velocity on the density is not as large. In the Chevalier self-similar solution, the radius of the shock wave depends on the ambient density (or the parameter $\dot{M} / v_{w}$ ) only as $\mathrm{R} \sim\left(\dot{M} / v_{w}\right)^{-1 /(n-s)}$, where $n$ is the power-law of the ejecta density profile and $s$ is that of the circumstellar medium. For $n=10$ and $s=2$ we get $\mathrm{R} \sim\left(\dot{M} / v_{w}\right)^{-1 / 8}$. Thus the higher density does not lead to significantly reduced velocities. Unfortunately these sources are even less frequent than IIb SNe. SN IIP form the most frequent type of SN (more than 50\% of the core-collapse SNe). These arise from RSG stars, and should theoretically have wind mass-loss rates ranging from $10^{-7}$ to $10^{-4}$ solar masses per year. However, observationally we find that most IIPs appear to arise from lower mass stars [30] and are less luminous in X-rays [31], suggesting on average a lower mass loss rate to wind velocity ratio. A case by case study is mandatory in these cases before any detailed predictions can be made. Finally, the equally rare $\mathrm{Ib} / \mathrm{Ic} \mathrm{SNe}$ are potentially interesting as they harbor the fastest shock waves, but they are usually associated with 
a Wolf-Rayet phase in the later phases of the massive star lifetime, that have a reasonably large mass-loss rate, but a wind velocity two orders of magnitude greater than RSGs, and therefore a correspondingly lower wind density, which tends to delay the peak of gamma-ray emission. Again a detail investigation of this class is warranted.

\section{Conclusion}

The highest energies in the CR spectrum require special environments to be produced. In agreement with the confinement criterium multi-PeV energies are to be either produced in large accelerators like massive star cluster or superbubbles [3, 4, 5] or in sites with high magnetic fields. The latter are found in young SNRs or in radio $\mathrm{SNe}$ (see T09 and [6]). In this work we argue that radio $\mathrm{SNe}$ could produce multi-PeV particles through the combination of favorable effects: fast shocks with velocities about $10 \%$ of the speed of light, high density CSM as produced by the wind of RSG stars and low wind magnetizations. A high ionization degree of the CSM would also ease the particle acceleration process. Under the condition that the background magnetic field has a turbulent component that prevents it from being purely toroidal, we have shown that the different instabilities driven by the acceleration of energetic particles could grow over intra-day timescales. If there is still some discussion about the exact way the magnetic fields can saturate and about the properties of the turbulence in the configuration of such fast shocks one can argue that protons with energies 1-10 possibly up to $50 \mathrm{PeV}$ can be accelerated within a few weeks after the SNe outburst. This model is also consistent with the magnetic field strengths deduced from the radio observations of the most luminous objects like SN 1993J (T09). Considering the fiducial case of SN 1993J, we have shown using a simple acceleration one-zone model that gamma-rays produced by pion decay could be easily detectable by CTA once the source become optically thin to pair production, i.e. about 7 days after the outburst. The predicted high-energy neutrino flux above 10 $\mathrm{TeV}$ from a SN 1993J type event is only about 0.1 neutrino for an instrument like Km3NeT. We have checked that the radio and $\mathrm{X}$-ray fluxes from the secondaries are consistent with the data. Our scenario suggests the possibility to produce clear predictions and hence to be testable by the forthcoming high-energy instruments. We have also discussed other types of SNe, and identified IIn SNe as possible strong gamma-ray emitters. But a full investigation of this possibility deserves further work. This work investigates a small parameter space of the SN forward shock properties and hence cannot address the important question of the formation of the CR spectrum (see the discussion in [32]). This aspect will also be addressed in a forthcoming work.

\section{References}

[1] Blasi, P., 2013, A\&ARv, 21, 710

[2] Hillas, A.M., 1984, ARA\&A, 22, 425

[3] Bykov, A., 2001, SSR, 99, 317

[4] Parizot, E., Marcowith, A., van der Swaluw, E., Tatischeff, V. \& Bykov A., 2004, A\&A, 424, 747

[5] Ferrand, G. \& Marcowith, A., 2010, A\&A, 510, 101

[6] Bell, A. R., Schure, K. M., Reville, B., Giacinti, G., 2013, MNRAS, 431, 415

[7] Tatischeff, V., 2009, A\&A., 499, 191 (T09)

[8] Maund J.R. et al., 2004, Nature, 427, 129

[9] Fransson C., Lunqvist, P. \& Chevalier, R.A., 1996, ApJ, 461, 933

[10] Parizot, E., Marcowith, A., Ballet, J. \& Gallant, Y., 2006, A\&A, 453, 387

[11] Marcowith A. \& Casse, F., 2010, A\&A, 515, 90

[12] Fransson C. \& Björnsson C.-I., 1998, ApJ, 509, 861

[13] Berezhko, E. \& Ellison, D.C., 1999, ApJ, 526, 385

[14] Bykov, A., Ellison, D.C. \& Renaud, M., 2012, SSR, 166, 71

[15] Bell, T. \& Lucek, S.G., 2000, MNRAS, 321, 433

[16] Bell, A. R., 2004, MNRAS, 353, 550

[17] Chandra, P., Ray, A., \& Bhtanagar, S. 2004, ApJ, 604, L97

[18] Vlemmings, W. H. T., IAU symposium, 242, 37

[19] Amato, E. \& Blasi, P., 2009, MNRAS, 392, 1591

[20] Pelletier, G., Lemoine, M. \& Marcowith, A., 2006, A\&A, 453, 181

[21] Bykov, A. M., Osipov, S. M., Ellison, D. C., MNRAS, 410, 39

[22] Dwarkadas, V., 2007, ApJ, 667, 226

[23] Beresnyak, A., Jones, T. W., Lazarian, A., 2009, ApJ, 707, 1541

[24] Giacalone, J. \& Jokipii, R., 2007, 663, L41

[25] Rogachevskii, I., Kleeorin, N., Brandenburg, A., Eichler, D., 2012, ApJ, 753, 6

[26] Zirakashvili, V. N., Ptuskin, V. S., Vlk, H. J., 2008, ApJ, 678, 255

[27] Lewis J.R. et al., 1994, MNRAS, 266, L27

[28] Weiler K.W. et al., 2007, 671, 1959

[29] Zimmermann H.-U. et al., 1994, Nature, 367, 621

[30] Smartt R.J., 2009, ARA\&A, 47, 63

[31] Dwarkadas V., 2014, MNRAS, 440,1917

[32] Ptuskin V.S, Zirakashvili, V.N., Seo, E.-S., 2010, ApJ, 718, 31 